significance to enhance the monitoring of Prorocentrum donghaiense ( $P$. donghaiense), a common red-tide species in China's coastal areas [18]. From the perspective of particles, this paper introduces the principles of experimental design, and sets up an ultrasonic testing system. The acoustic backscattering signals of $P$. donghaiense at different concentrations were acquired in the lab. Then, the relationship between acoustic signals and algae cell concentrations were determined through analysis, and subjected to descriptive verification, leading to meaningful conclusions.

\section{EXPERIMENTAL PRINCIPLES}

\subsection{Basic ultrasonic scattering features of particles}

During the propagation in particle-containing medium, the incident ultrasound wave may change direction due to the presence of particles, which does not occur in the traditional continuous medium. When a plane wave reaches a particle, part of it will continue to propagate forward, and another part of it will be scattered by the particle to the surrounding or backwards. Both parts contain the information on the scatterers.

The scattering behavior of the particle is determined by the ratio of particle size and wavelength. The dimensionless expression of the wave number can be expressed as:

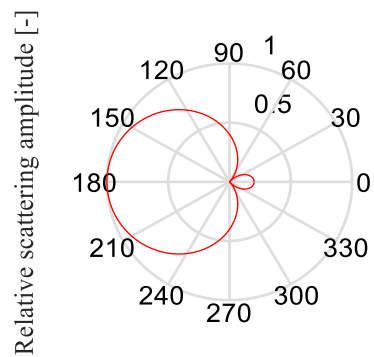

(a) Scattering angle $\left[{ }^{\circ}\right]$

$$
k a=\frac{2 \pi}{\lambda} \cdot \frac{d}{2}=\frac{\pi \cdot d}{\lambda}=\frac{\pi \cdot f \cdot d}{c}=\frac{2 \pi \cdot f \cdot a}{c}
$$

where, $d$ is the particle diameter; $a$ is the particle radius; $f$ is the acoustic frequency; $\lambda$ is the wavelength.

Considering the scattering in the Rayleigh model [19, 20], the scattering intensity of the incident plane wave by a single hard particle at the angle $\theta$ corresponding to the far-field distance $r$ can be defined simply as:

$$
\begin{gathered}
I_{S}(r, \theta)=\frac{p_{0}^{2}}{2 \rho_{0} c_{0}} \cdot \frac{a^{2}}{r^{2}}|R(\theta)|^{2}=I_{0} \frac{a^{2}}{r^{2}} \cdot|R(\theta)|^{2}=I_{0} \frac{a^{2}}{r^{2}} . \\
\frac{1}{(k a)^{2}} \sum_{l, n=0}^{\infty}\left[b_{l} b_{n}^{*} e^{j \frac{l-n}{2} \pi} P_{l}(\cos \theta) P_{n}(\cos \theta)\right]
\end{gathered}
$$

where, $|R(\theta)|^{2}$ is the squared value of the absolute value (modulus) of the complex number $R(\theta)$. This complex number stands for the directivity of the scattering sound field:

$$
\begin{gathered}
R(\theta)=\frac{1}{k a} \sum_{l=0}^{\infty} b_{l} e^{j \frac{l+1}{2} \pi} P_{l}(\cos \theta)=\frac{1}{k a} \sum_{l=0}^{\infty}(-j)^{l}(2 l+ \\
\text { 1) } \frac{\frac{d\left[j_{l}(k a)\right]}{d(k a)}}{\frac{\left.d h_{l}^{2}(k a)\right]}{d(k a)}} \cdot e^{j \frac{l+1}{2} \pi} P_{l}(\cos \theta)
\end{gathered}
$$

where, $P_{l}(\cos \theta)$ is the Legendre function; $j_{l}(k a)$ is the spherical Bessel function; $h_{l}^{2}(k a)$ is the Hankel spherical function of the second kind; $a$ is the particle radius.

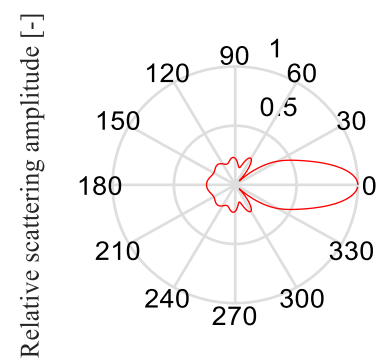

(b) Scattering angle $\left[{ }^{\mathrm{o}}\right]$

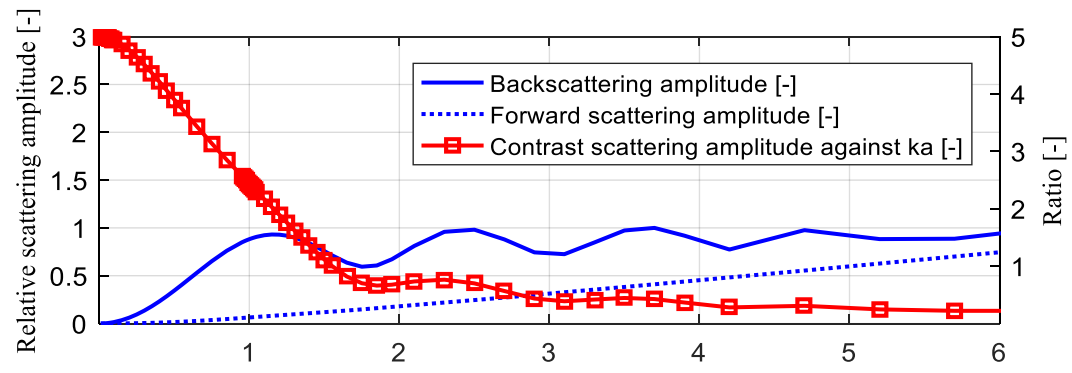

(c) Dimensionless wave number ka [-]

Figure 1. Polar coordinate profiles of particle acoustic scattering (the variation of normalized scattering amplitude with scattering angles) (a) Particle scattering profile of $k a=0.03$, (b) Particle scattering profile of $k a=6$, (c) The polar coordinates ratio of backscattering to forward scattering as a function of $k a$

Figure 1 shows the polar coordiante profiles of particle acoustic scattering at different $k a$ values. It can be seen that scattering amplitude differs in the range of the $360^{\circ}$ scattering angle. Then, the acoustic scattering intensity was normalized. The scattering in the $0^{\circ}$ direction, which coincides with the direction of the incident wave, was defined as forward scattering; the scattering in the opposite direction was defined as backscattering, the scattering in the $180^{\circ}$ direction, the directivity was expressed as both forward scattering and backscattering.
It can be seen from Figure 1(a) that the backscattering was relatively uniform when the $1 \mathrm{MHz}$ incident acoustic wave reached a $14 \mu \mathrm{m}$ particle $(k a \approx 0.03)$, and much greater than forward scattering. With the increase of the $k a$, the intensity of the scattered acoustic waves exhibited an obvious non-uniform spatial distribution, and the number of petals grew in the scattering plot. After the $k a$ increased to a certain value, the backscattering underwent a sharp decline. As shown in Figure 1(b) $(k a \approx 6)$, the scattered sound waves mainly moved forward, and interfered with the incident wave, forming a prominent 
geometric shaded area.

According to Figure 1(c), both backscattering and forward scattering were strengthened with the increase of $k a$. Specifically, the backscattering fluctuated obviously near $k a=1$ and then gradually became stable (solid curve), while the forward scattering always increased with the value of $k a$ (dashed curve). In addition, the polar coordinates ratio of backscattering to forward scattering (dotted curve) is inversely proportional to $k a$ : the ratio was relatively large at a small $k a$, and decreased gradually to zero with the growth of $k a$.

Because of the huge number of microalgae (particles) being detected, it is feasible to detect the backscattering signals with a single transmit-receive (T-R) probe, although backscattering intensity of a single particle has a small absolute value. In other words, it is reasonable to collect the backscattering signals of microalgae with a particle size of micron using an $\mathrm{MHz}$ ultrasound probe $(k a \ll 1)$

\subsection{Equivalent estimation of particle backscattering intensity}

To estimate the particle backscattering intensity, it is assumed that the plural scattering is so small as negligible, the particles exist as individuals in the solution, and the $P$. donghaiense cells are equivalent to spherical particles with a certain hardness. Since algae particles exist as fluid spheres, $\sigma$, the theoretical backscattering cross section of a single cell can be calculated as:

$$
\sigma=k^{4} a^{6}\left[\frac{1-g h^{2}}{3 g h^{2}}+\frac{1-g}{1+2 g}\right]^{2}
$$

where, $g$ is the density ratio of algae cell to the seawater medium; $h$ is the sound velocity ratio of algae cell to the seawater medium. The two parameters cannot be determined without advanced test devices. Here, their values are both estimated as 1.05 according to the measured results in the existing literature [16].

Then, the total volume backscattering strength $S_{\mathrm{v}}$ can be obtained by accumulating the backscattering intensity of each particle:

$$
S_{\mathrm{v}}=10 \log _{10}(\mathrm{~N} \sigma)+\mathrm{C}_{\mathrm{e}}
$$

where, $N$ is the number of particles in the seawater medium; $\mathrm{C}_{\mathrm{e}}$ is the environmental factor.

While it is difficult to measure the exact backscattering intensity through experiment, there is a certain correspondence between the integrated backscatter strength (IBS), and the theoretical total volume backscattering strength $S_{v}$ :

$$
\text { IBS }=20 \log _{10}\left(\frac{V_{\text {rms }} B s}{V_{\text {rms }} \text { Ref }}\right)
$$

where, $\mathrm{V}_{\mathrm{rms}} \mathrm{Bs}$ is the effective voltage of the backscattering signals in the seawater containing a certain concentration of $P$. donghaiense; $\mathrm{V}_{\mathrm{rms}} \mathrm{Ref}$ is the effective voltage of the backscattering signals in the pure seawater (the reference medium); rms is short for root mean square. Therefore, the number of particles can be estimated through comparative analysis on the acoustic and electrical signals of algae solutions at different concentrations.

\section{CONSTRUCTION} PLATFORM

\subsection{Preparation of algae solutions}

P. donghaiense is an earthy yellow, heliophilous single-cell

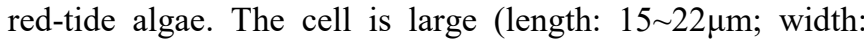
9 14 $\mu \mathrm{m}$ ), and shaped like an asymmetrical pear, with a slight dent on the top. Most cells have round bottoms and long flagella [21]. The aseptic strains of the $P$. donghaiense solutions at different concentrations were provided by State Key Laboratory of Marine Environmental Science (Xiamen University).

The seawater was collected from the sea to the west of Xiamen, filtered through a cellulose acetate membrane $(0.2 \mu \mathrm{m})$ to remove microalgae and particulate matters, and subjected to autoclave sterilization. Next, the $\mathrm{pH}$ and salinity of the seawater were adjusted to $8.0 \pm 0.2$ and $30 \mathrm{psu}$, respectively. After that, the seawater was enriched by sterilized $\mathrm{f} / 2$ nutrient solution [22]. The nutritive salts and vitamins of the $P$.

\begin{tabular}{|c|c|c|}
\hline Compound & Quantity & Stock solution \\
\hline \multicolumn{3}{|c|}{ (a) $\mathrm{f} / 2$ medium, added to $950 \mathrm{~mL}$ filtered seawater } \\
\hline $\mathrm{NaNO}_{3}$ & $1 \mathrm{~mL}$ & $75 \mathrm{~g} / \mathrm{L} \mathrm{dH} 2 \mathrm{O}$ \\
\hline $\mathrm{NaH}_{2} \mathrm{PO}_{4} \cdot \mathrm{H}_{2} \mathrm{O}$ & $1 \mathrm{~mL}$ & $5 \mathrm{~g} / \mathrm{L} \mathrm{dH_{2 } \mathrm { O }}$ \\
\hline $\mathrm{Na}_{2} \mathrm{SiO}_{3} \cdot 9 \mathrm{H}_{2} \mathrm{O}$ & $1 \mathrm{~mL}$ & $30 \mathrm{~g} / \mathrm{L} \mathrm{dH} \mathrm{H}_{2} \mathrm{O}$ \\
\hline $\begin{array}{l}\mathrm{f} / 2 \text { trace metal } \\
\text { solution }\end{array}$ & $1 \mathrm{~mL}$ & (b) \\
\hline $\mathrm{f} / 2$ vitamin solution & $1 \mathrm{~mL}$ & (c) \\
\hline \multicolumn{3}{|c|}{ (b) $\mathrm{f} / 2$ trace metal solution, added to $950 \mathrm{~mL} \mathrm{dH}_{2} \mathrm{O}$} \\
\hline $\mathrm{FeCl}_{3} \cdot 6 \mathrm{H}_{2} \mathrm{O}$ & $3.15 \mathrm{~g}$ & \\
\hline $\mathrm{Na}_{2} \mathrm{EDTA} \cdot 2 \mathrm{H}_{2} \mathrm{O}$ & $4.36 \mathrm{~g}$ & \\
\hline $\mathrm{CuSO}_{4} \cdot 5 \mathrm{H}_{2} \mathrm{O}$ & $1 \mathrm{~mL}$ & $9.8 \mathrm{~g} / \mathrm{L} \mathrm{dH}_{2} \mathrm{O}$ \\
\hline $\mathrm{Na}_{2} \mathrm{MoO}_{4} \cdot 2 \mathrm{H}_{2} \mathrm{O}$ & $1 \mathrm{~mL}$ & $6.3 \mathrm{~g} / \mathrm{L} \mathrm{dH} 2 \mathrm{O}$ \\
\hline $\mathrm{ZnSO}_{4} \cdot 7 \mathrm{H}_{2} \mathrm{O}$ & $1 \mathrm{~mL}$ & $22.0 \mathrm{~g} / \mathrm{L} \mathrm{dH} 2 \mathrm{O}$ \\
\hline \multicolumn{3}{|c|}{ (c) $\mathrm{f} / 2$ vitamin solution, added to $950 \mathrm{~mL} \mathrm{dH}_{2} \mathrm{O}$} \\
\hline $\begin{array}{c}\text { Vitamin } \mathrm{B}_{12} \\
\text { (cyanocobalamin) }\end{array}$ & $1 \mathrm{~mL}$ & $1.0 \mathrm{~g} / \mathrm{L} \mathrm{dH} \mathrm{H}_{2} \mathrm{O}$ \\
\hline Biotin & $1 \mathrm{~mL}$ & $0.1 \mathrm{~g} / \mathrm{L} \mathrm{dH_{2 }} \mathrm{O}$ \\
\hline Thiamine $\mathrm{HCl}$ & $200 \mathrm{mg}$ & \\
\hline
\end{tabular}
donghaiense culture solutions are listed in Table 1 below.

Table 1. Compounds of $P$. donghaiense culture media

The initial inoculation and culturing were carried out in a $250 \mathrm{~mL}$ conical flask, containing $180 \mathrm{~mL}$ fresh $\mathrm{f} / 2$ culture solution, in a special sterile culture room at the temperature of $21^{\circ} \mathrm{C}$, the light-dark cycle of $12 \mathrm{~h}: 12 \mathrm{~h}$, and the light intensity of $70 \mu \mathrm{mol} /\left(\mathrm{m}^{2} \cdot \mathrm{s}\right)$. The flask was shaken twice a day to prevent precipitation and wall growth. After activation and repeated transfers, the number of $P$. donghaiense cells grew steadily. The number increased exponentially from the $2^{\text {nd }}$ to the $4^{\text {th }}$ day and stably from the $6^{\text {th }}$ to the $8^{\text {th }}$ day.

The observation was carried out using a Type B LISST-100 laser in situ particle size analyzer, and the results were recorded in Figure 2. It can be seen that the particle sizes of $P$. donghaiense cells mainly concentrated between $8 \mu \mathrm{m}$ and $22 \mu \mathrm{m}$. The equivalent particle size $D_{60}$ of the $P$. donghaiense in our experiment was measured as $14 \mu \mathrm{m}$, about $5 \%$ smaller on average than the general size. This means the wavelength of $1 \mathrm{MHz}$ ultrasound far exceeds the particle size of $P$. donghaiense cells $(k a \ll 1)$, which satisfies the premise of the Rayleigh scattering model.

The $P$. donghaiense solutions at different concentrations were prepared from algae cell particles in pure seawater, the 
algae solutions were collected in the late phase of the exponential growth period for testing. The direct detection of the water samples show that the number of cells in each algae solution is as follows: $0,70,120,2.38 \times 10^{2}, 4.3 \times 10^{2}$, $7.6 \times 10^{2}, 9.6 \times 10^{2}, 1.9 \times 10^{3}, 4.1 \times 10^{3}, 9.4 \times 10^{3}, 1.5 \times 10^{4}$, $2.4 \times 10^{4}$ and $5.6 \times 10^{4} \mathrm{cell} / \mathrm{mL}$.

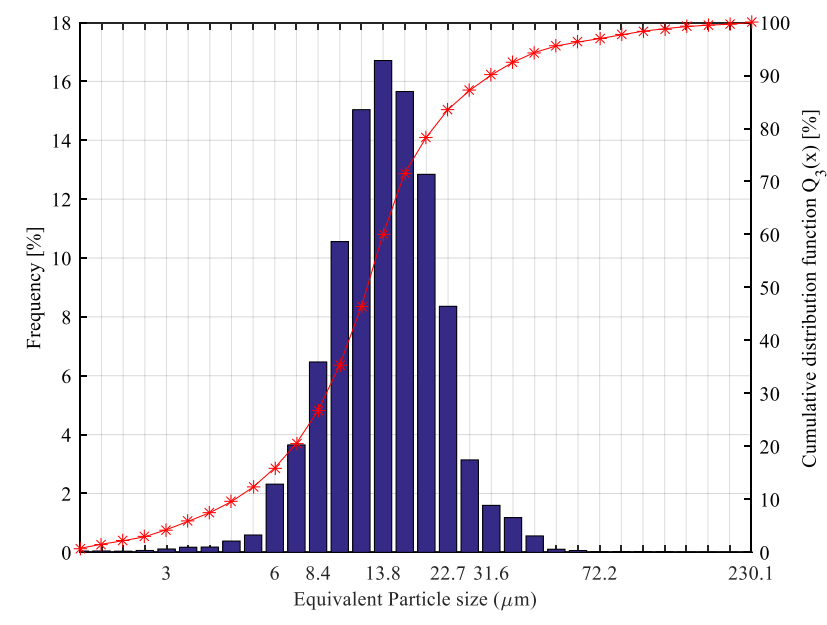

Figure 2. Particle size distribution of $P$. donghaiense

\subsection{Platform construction}

Traditionally, the solution features are characterized by the attenuation of the ultrasound transmitting through the medium between two opposite acoustic probes. In our experiment, however, a single immersion ultrasonic T-R probe (nominal central frequency: $1 \mathrm{MHz}( \pm 30 \mathrm{kHz})$; half-power angle: $6^{\circ}$; sharpness angle: $14^{\circ}$; outer diameter: $8 \mathrm{~mm}$ ) is adopted to measure the backscattering of the ultrasound. As shown in Figure 3, the measurement system of our experiment mainly consists of four modules: algae solution storage and testing, programmable circuit board, digital oscilloscope and computer data analysis. The test tank is a $5 \mathrm{~L}$ glass beaker with a height of $275 \mathrm{~mm}$ and an inner diameter of $180 \mathrm{~mm}$. The size of the tank is sufficient to meet the distance requirements for far-field acoustic measurement, because the probe has a small diameter and a high frequency. The measurements were performed at $23{ }^{\circ} \mathrm{C}$, and the room temperature was kept basically constant.

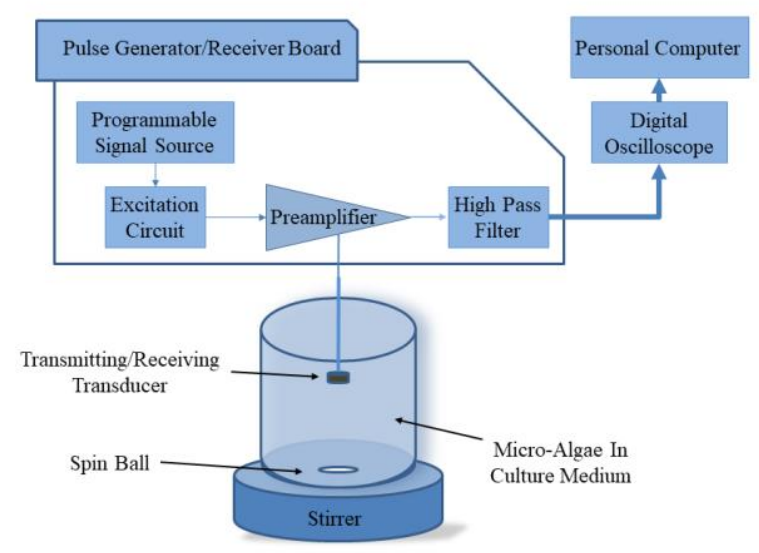

Figure 3. Block diagram of the measurement platform
The experiment was carried out through the following steps. Firstly, the transmitting signals for the algae solutions with pre-set parameters were written into the circuit system through the chip on the circuit board, and converted into ultrasound signals before being sent at the $\mathrm{T} / \mathrm{R}$ probe attached to the end of the circuit board. Then, the ultrasound signals propagated through algae solutions at different concentrations, and interacted with the algae cells. The feedback signals (backscattering signals) were received by the same probe, and converted into electrical signals. Then, the signals were amplified and filtered on the circuit board, sampled by the digital oscilloscope UT2102CE (the maximum sampling frequency: $1 \mathrm{GS} / \mathrm{s}$ ), and uploaded to the PC for data analysis, yielding the final results.

To prevent interference and superposition between transmitted signals, the probe excited a square wave pulse of $1 \mathrm{MHz}$ and sent it at the interval of $30 \mathrm{~s}$. For each concentration, the measurement was repeated 60 times and the result of each measurement were recorded. Moreover, the solutions were stirred magnetically at the bottom once every 20 measurements, aiming to eliminate the effect of solution unevenness caused by cell sedimentation and that of bubbles formed in agitation. After each stirring, the solution was left to stabilize for a short while before entering the next measurement. During the ultrasonic measurement, three parallel samples were prepared for each concentration, and the collected signals were subjected to mean processing and were cut into $20 \mu \mathrm{s}-$ long segments for PC processing.

\section{EXPERIMENTAL RESULTS}

A warning should be issued red tide once the $P$. donghaiense concentration reaches $5 \times 10^{5}$ cell/L [23,24]. Generally, low, medium and high concentrations are respectively defined as the concentration below, near and obviously higher than the warning level, and ultra-high concentration is defined as the concentration greater than the level at the outbreak of red tide. Focusing on red tide forecast, this paper mainly explores the acoustic features of algae at low and medium concentrations.

Ultrasonic measurements were performed on $P$. donghaiense sample solutions at 12 different concentrations. The measured results in Figure 4 show that the IBS (red solid dots) increased with the algae concentration.

The acoustic result of measured data (red dot) exhibited similar trend as that predicted by the Rayleigh theoretical model in Eq. (5) (red up arrows). Considering that $P$. donghaiense cells are poly-dispersed particles, the upper and lower bounds of particle size should be determined through theoretical calculation according to the particle size distribution features in Figure 2, and the main particle sizes should be selected for further correction by the following equation:

$$
\mathrm{S}_{\mathrm{vN}_{\mathrm{M}}}=10 \log _{10}\left(\sum_{\mathrm{i}=1}^{\mathrm{N}_{\mathrm{M}}} \mathrm{N}_{\mathrm{i}} \cdot \sigma_{\mathrm{i}}\right)+\mathrm{C}_{\mathrm{e}}
$$

where, $\mathrm{N}_{\mathrm{M}}$ is the number of main particle sizes being selected. Here, seven particles size $(8.4 \sim 22.7 \mu \mathrm{m})$ are selected, i.e. $\mathrm{N}_{M}=7$. For each particle size, the backscattering intensity $\sigma_{i}$ of the single particle was computed, and multiplied with the number of particles $\mathrm{N}_{\mathrm{i}}$. On this basis, the total backscattering intensity $\mathrm{S}_{\mathrm{VN}}$ was obtained by adding up the results of the seven particle sizes. The revised theoretical equation contains 
information on particle size and concentration, providing a reliable reference for actual measurements. Compared with the results calculated by equivalent particle size only, the theoretical values corrected by the actual particle sizes of $P$. donghaiense (red curve with boxes) enjoy high intensity and close proximity to the measured values, indicating that it is feasible to monitor algae concentration by measuring backscattering sound signals.

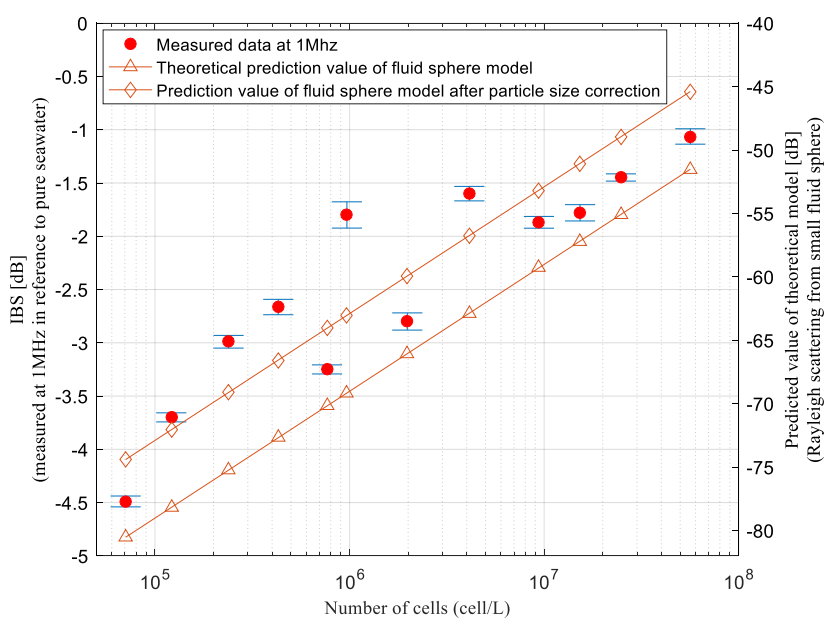

Figure 4. Measured results vs. theoretical predictions

\section{DISCUSSION}

\subsection{Results analysis and verification}

Through the lab acoustic measurements, the author acquired the basic acoustic features of backscattering intensity of $P$. donghaiense solutions at different concentrations, and derived similar linear trends with those predicted by the theoretical model. However, the theoretical model was simplified under the ideal condition. It can be seen from Figure 4 that the predicted result differed from the measured IBS in the rate of change.

In view of the above, the acoustic data of the 12 algae solutions were fitted in different concentration segments. As shown in Figure 5, the IBS increased much faster in the low and medium concentration segment (up arrows; $<9 \times 10^{5} \mathrm{cell} / \mathrm{L}$ ) than in the medium and high concentration segment (down arrows; $>4 \times 10^{6}$ cell $/ \mathrm{L}$ ). This difference can be attributed to the following factors: The IBS is very sensitive to the growth in particle concentration, when the solution is as weak as low concentration algae solutions; thus, the intensity increased rapidly with the rise of the particle concentration. By contrast, the intense interactions between particles in high concentration algae solutions hindered the transmission of backscattering soundwave, which drags down the growth rate of the IBS.

After that, the acoustic data of all solutions were fitted again with quadratic polynomial and the results $\left(\mathrm{R}^{2}=0.81\right)$ are drawn as a solid curve in Figure 5. It can be seen that the quadratic polynomial fitting achieved similarly good correlation with the linear fitting (dashed curve), and outperformed the latter in the correlation with the measured IBS in the two ends of high and low concentrations.

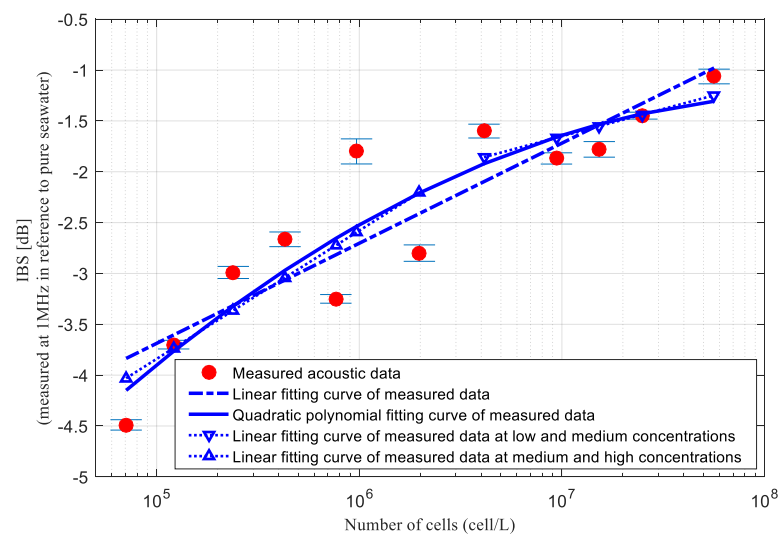

Figure 5. Measured acoustic features and the fitting results

The default equivalent particle size of $P$. donghaiense was $14 \mu \mathrm{m}$. The scattering can be viewed as uncorrelated when the particle spacing is greater than 3 times the particle size. The warning concentration falls within this range. However, the high concentration above this range has a certain acoustic attenuation effect in the sound path range, and needs to be corrected in two steps: collecting the ultrasound signals received in each test on algae solution at a specific concentration into a sample set, and finding the mean square value of the set; conducting exponential fitting of the outer envelope of the mean squared waveform, and expressing the outer envelope signals as:

$$
E_{i}(t)=E_{0} \cdot \exp \left(-\alpha_{i t} \cdot t\right)
$$

where, $E_{i}$ is the mean square value at the concentration $N_{i} ; t$ $(\mu \mathrm{s})$ is the duration variable of the observation window; $\alpha_{i t}$ $(\mathrm{dB} / \mu \mathrm{s})$ is the attenuation coefficient for the exponential fitting of the outer envelope. Note that the scattering attenuation coefficient $\alpha_{s}=\alpha_{t} / C(\mathrm{~dB} / \mathrm{cm})$, with $C$ being the sound velocity. Without considering the effects of particle concentration in the medium, the sound velocity is basically stable at the same conductivity, temperature and depth. Taking the logarithm on both sides, the sound attenuation effect can be linearized.

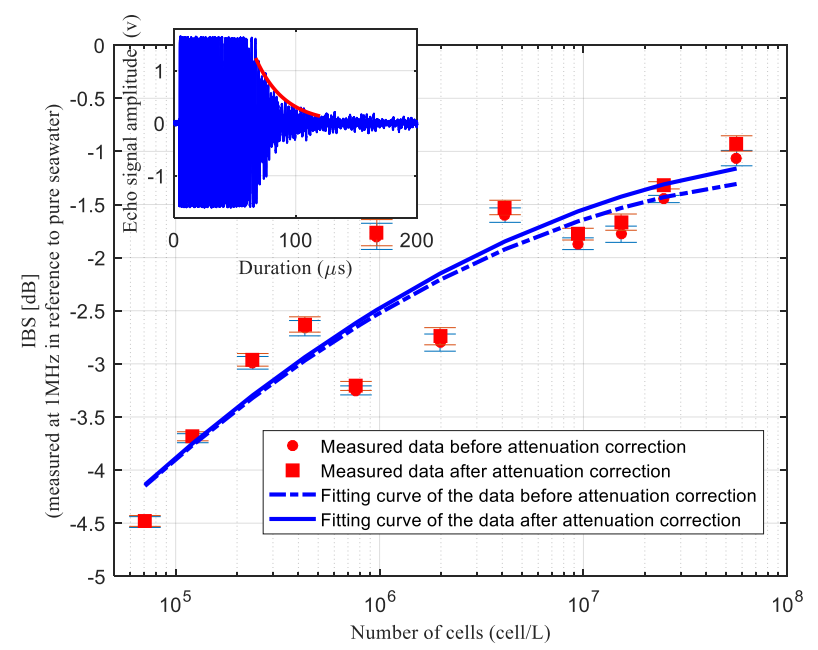

Figure 6. Pre- and post-correction IBS values 
Figure 6 compares the IBS values of $P$. donghaiense solutions at different concentration before and after attenuation correction. The post-correction measured data are presented as red boxes. It can be seen that the IBS values of low concentration algae solutions increased linearly with the number of algae cells, indicating that the attenuation is rather weak. The IBS values of medium concentration algae solutions fluctuated with the increase of concentration, revealing a gradual enhancement of the attenuation. The attenuation was compensation in high concentration algae solutions, because the growth in backscattering was suppressed by the obvious attenuation. After the compensation, the IBS values (solid curve) were greater than the uncompensated values (dashed curve), and the linear features were improved. The correlations of both linear and nonlinear fittings were enhanced.

New $P$. donghaiense solutions were prepared at another six concentrations: $1.92 \times 10^{2}, 3.6 \times 10^{2}, 8.1 \times 10^{2}, 1.17 \times 10^{3}$, $2.49 \times 10^{3}$ and $6.35 \times 10^{3} \mathrm{cell} / \mathrm{mL}$, the results are shown in Figure 7 below.

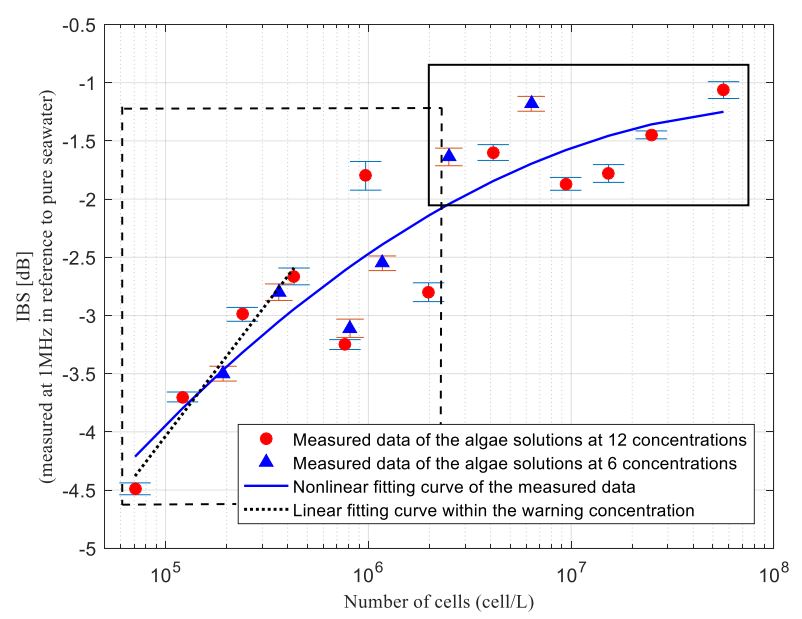

Figure 7. Measured data vs. fitting curves

It can be seen that the IBS values (blue up arrows) of the new $P$. donghaiense solutions at the six concentrations conformed to the fitting curve (red dots) of the predictions at the 12 concentrations in the basic test, showing a positive correlation with algae concentration. The deviation from the original fitting curve fell within the allowable range. Comparing the results of all samples in the two tests, it is learned that the correlation between the IBS and concentration was weak and fluctuating in the medium concentration segment. However, the IBS and concentration had an obvious linear correlation in the low and medium concentration segment (dashed box), especially within the warning concentration $5 \times 105 \mathrm{cell} / \mathrm{L} \quad(\mathrm{R} 2=0.96)$. In the high concentration segment (solid box), the correlation was also highly linear, for the linear growth rate was suppressed by the attenuation effect of high concentration algae cells on sound propagation. The above results show that our test method is feasible and may apply to other concentrations.

\subsection{Uncertainty analysis}

The uncertainty components of our measurement platform come from repeated measurements (type A) and defects of the platform or measurement method (type B) [25]. Type A uncertainty $U_{A}$ can be obtained by computing the statistical average of multiple independent measurements on the algae samples:

$$
U_{A}=S D_{n}(\bar{\alpha})=\sqrt{\frac{1}{n(n-1)} \sum_{i=1}^{n}\left(\alpha_{i}-\bar{\alpha}\right)^{2}}
$$

where, $\mathrm{S} D_{n}(\bar{\alpha})$ is the standard deviation of the average IBS. Table 2 lists the Type A uncertainties at different algae concentrations. It can be seen that the algae solution at $9.6 \times 10^{5}$ cell/L had the greatest uncertainty.

Type B uncertainty components of measurement platform may come from the inconsistency of probe position, the deviation from electrical noise of the measuring instrument, the varied distributions of particle size, the change in experimental temperature, and the inaccurate measurement of solution volume. For our measurement platform, temperature change and volume inaccuracy are the main causes of type B uncertainties. This is because our tests use the same set of circuit system and the same probe, and the algae solutions were prepared after shaking and tested with magnetic stirring and standing. Here, it is assumed that the measurement errors of solution volume and temperature are $\pm 1 \%$ and $\pm 0.5^{\circ} \mathrm{C}$, respectively.

During the analysis of type $\mathrm{B}$ uncertainties, $t^{+}$and $t^{-}$are the IBS values for each $0.5{ }^{\circ} \mathrm{C}$ increase and decrease of temperature, respectively; $V^{+}$and $V^{-}$are the IBS values for each $1 \%$ of increase and decrease of volume, respectively; $\mathrm{ARD} / \mathrm{t}$ and $\mathrm{ARD} / \mathrm{V}$ are the average relative deviations of the IBS induced by volume change and temperature change, respectively. The IBS was measured at $23{ }^{\circ} \mathrm{C}(\mathrm{dB})$. The type B uncertainty $u_{B}$ can be calculated as:

$$
u_{B}=\frac{1}{2} \sqrt{u_{B t}^{2}+u_{B v}^{2}}
$$

The total uncertainty $U$ can be computed as:

$$
U=k \sqrt{u_{A}^{2}+u_{B}^{2}}
$$

where, $k=2$. The type $\mathrm{B}$ uncertainties of backscattering coefficients in algae solutions at different concentrations are displayed in Table 3 below. Obviously, the algae solution at $9.6 \times 10^{5} \mathrm{cell} / \mathrm{L}$ had the greatest uncertainty, which is basically the same with the result of Figure 7 (the deviation was relatively large at $8.1 \times 10^{5}$ cell $/ \mathrm{L}$ ). Hence, the maximum measurement uncertainty of our measurement system was determined as $23.22 \%$

Table 2. Type A uncertainties of backscattering coefficients in algae solutions at different concentrations

\begin{tabular}{|c|c|c|c|c|c|c|c|c|}
\hline$/\left(\times 10^{5}\right.$ cell $\left.\cdot L^{-1}\right)$ & \multicolumn{9}{|c|}{$1 \mathrm{MHz}$} \\
\hline Concentration & 0.7 & 1.2 & 1.9 & 2.38 & 3.6 & 4.3 & 7.6 & 8.1 \\
\hline Type A uncertainty & $4.33 \%$ & $3.61 \%$ & $5.42 \%$ & $5.06 \%$ & $6.03 \%$ & $6.14 \%$ & $3.61 \%$ & $6.68 \%$ \\
\hline Concentration & 9.6 & 11.7 & 19 & 41 & 94 & 152 & 248 & 564 \\
\hline Type B uncertainty & $10.48 \%$ & $5.35 \%$ & $6.86 \%$ & $5.78 \%$ & $4.7 \%$ & $6.5 \%$ & $2.89 \%$ & $6.14 \%$ \\
\hline
\end{tabular}


Table 3. Type B uncertainties of backscattering coefficients in algae solutions at different concentrations

\begin{tabular}{|c|c|c|c|c|c|c|c|c|}
\hline Type B uncertainties & \multicolumn{7}{|c|}{ Concentration/ $\left(\times 10^{5} \mathrm{cell} \cdot \mathrm{L}^{-1}\right)$} \\
\cline { 2 - 8 } & 1.9 & 3.6 & 4.3 & 7.6 & 8.1 & 9.6 & 11.7 & 19 \\
\hline IBS (dB) & -3.501 & -2.809 & -2.661 & -3.253 & -3.114 & -1.802 & -2.553 & -2.807 \\
\hline$t^{+}$ & -3.469 & -2.732 & -2.614 & -3.231 & -3.082 & -1.690 & -2.484 & -2.722 \\
\hline$t^{-}$ & -3.560 & -2.877 & -2.736 & -3.300 & -3.176 & -1.942 & -2.624 & -2.896 \\
\hline $\mathrm{ARD} / t$ & $1.43 \%$ & $2.64 \%$ & $2.42 \%$ & $1.21 \%$ & $1.71 \%$ & $7.25 \%$ & $2.78 \%$ & $3.21 \%$ \\
\hline$V^{+}$ & -3.563 & -2.876 & -2.745 & -3.294 & -3.184 & -1.933 & -2.638 & -2.890 \\
\hline$V^{-}$ & -3.468 & -2.736 & -2.605 & -3.229 & -3.066 & -1.692 & -2.496 & -2.723 \\
\hline $\mathrm{ARD} / V$ & $1.50 \%$ & $2.57 \%$ & $2.76 \%$ & $1.12 \%$ & $2.05 \%$ & $6.89 \%$ & $2.98 \%$ & $3.05 \%$ \\
\hline$u_{B}$ & $1.04 \%$ & $1.84 \%$ & $1.83 \%$ & $0.83 \%$ & $1.33 \%$ & $5.00 \%$ & $2.04 \%$ & $2.21 \%$ \\
\hline$U$ & $11.03 \%$ & $12.62 \%$ & $12.82 \%$ & $7.41 \%$ & $13.63 \%$ & $23.22 \%$ & $11.44 \%$ & $14.42 \%$ \\
\hline
\end{tabular}

\section{CONCLUSIONS}

In this paper, an acoustic measurement platform is designed and adopted for the ultrasonic measurement of $P$. donghaiense solutions at different concentration. The following conclusions were put forward after analyzing the measured results:

(1) The proposed single-probe acoustic measurement platform for $P$. donghaiense solutions can capture the backscattering sound signals of algae solutions at different concentrations. The IBS value gradually increased as the concentration rose from $1.92 \times 10^{5}$ to $6.35 \times 10^{6} \mathrm{cell} / \mathrm{L}$. This correlation agrees well with the theoretical results. The prediction effect can be further improved through the particle size correction of the simplified theoretical formula.

(2) The IBS and concentration had an obvious linear correlation when the $P$. donghaiense concentration was below $1 \times 10^{6}$ cell/L, especially within the warning concentration $5 \times 10^{5} \mathrm{cell} / \mathrm{L}$. This relationship satisfies the application scope and requirements of red tide forecast. With the growth in concentration, the linear correlation between the IBS and concentration weakened and fluctuated. After further increase of concentration, the linear growth rate and amplitude of the IBS were suppressed by the attenuation effect of high concentration algae cells on sound propagation.

(3) Within the concentration range of $P$. donghaiense in our experiment, the IBS value exhibited a linear growth trend with the increase in concentration. Considering the attenuation effect, the IBS variation was discussed in three phases. For high concentration sampling points, the attenuation effect was mitigated through compensation, thus improving the correlation between the IBS and concentration. The measured data were fitted by quadratic polynomial, and the fitting curves were divided into different segments. Then, the measurement platform was improved through comparison between the different segments.

(4) Despite some uncertainties in the experiment, the experiment process is generally reasonable. Thus, the proposed platform can be applied to monitor the solutions at other concentrations. The future research will probe deeper into the accuracy of the measurement.

To sum up, it is feasible and effective to measure the concentration of $P$. donghaiense by ultrasound. With high cost-effectiveness, the proposed measurement approach supports real-time online monitoring. The research findings provide valuable references to the observation and monitoring of red tides in China, shed new light on red tide forecast, prevention and control, and promote marine ecology and coastal economy.

\section{REFERENCES}

[1] Kudela, R., Berdalet, E., Enevoldsen, H., Pitcher, G., Raine, R., Urban, E. (2017). GEOHAB-the global ecology and oceanography of harmful algal blooms program: motivation, goals, and legacy. Oceanography, 30(1): 12-21. https://doi.org/10.5670/oceanog.2017.106

[2] Landsberg, J.H. (2002). The effects of harmful algal blooms on aquatic organisms. Reviews in Fisheries Science, $10(2)$ : 113-390. https://doi.org/10.1080/20026491051695

[3] Hallegraeff, M. (2010). Ocean climate change, phytoplankton community responses, and harmful algal blooms: A formidable predictive challenge1. Journal of Phycology, 46(2): 220-235. https://doi.org/10.1111/j.1529-8817.2010.00815.x

[4] Anderson, D.M., Andersen, P., Bricelj, V.M., Cullen, J.J., Rensel, J.E. (2001). Monitoring and management strtegies for harmful algal blooms in coastal waters. Unesco, 2001.

[5] Aliotta, G., Feo, D.V., Pinto, G., Pollio, A. (1999). In vitroinhibition of algal growth byrut a graveolensl. extracts: Biological and chemical aspects. Plant Biosystems. An International Journal Dealing with All Aspects of Plant Biology, 133(2): 185-191. https://doi.org/10.1080/11263509909381547

[6] Terlizzi, D.E., Ferrier, M.D., Armbrester, E.A., Anlauf, K.A. (2002). Inhibition of dinoflagellate growth by extracts of barley straw (Hordeum vulgare). Journal of Applied Phycology, 14(4): 275-280. https://doi.org/10.1023/a:1021164302634

[7] Jiang, D.L., Ni, G.W., Zhang, Y.M., Su, Y.P. (2012). Algal control by low-frequency, low-power ultrasonic in eutrophic water bodies. Advanced Materials Research, 433-440:

811-816.

https://doi.org/10.4028/www.scientific.net/amr.433440.811

[8] Ahn, Y.H., Shanmugam, P., Chang, K.I., Moon, J.E., Ryu, J.H. (2005). Spatial and temporal aspects of phytoplankton blooms in complex ecosystems off the korean coast from satellite ocean color observations. Ocean Science Journal, 40(2): 67-71. https://doi.org/10.1007/bf03028587

[9] Stumpf, R.P. (2001). Applications of satellite ocean color sensors for monitoring and predicting harmful algal blooms. Human and Ecological Risk Assessment: An International Journal, 7(5): 1363-1368. https://doi.org/10.1080/20018091095050

[10] Stanton, T.K., Wiebe, P.H., Chu, D. (1998). Differences between sound scattering by weakly scattering spheres 
and finite-length cylinders with applications to sound scattering by zooplankton. The Journal of the Acoustical Society of America, 103(1): 254-264. https://doi.org/10.1121/1.421135

[11] Frisk, G.V., Hays, E.E. (1978). Acoustical oceanography, principles and applications. Deep Sea Research, 25(8): 749. https://doi.org/10.1016/0146-6291(78)90629-x

[12] Holliday, D.V., Pieper, R.E. (1980). Volume scattering strengths and zooplankton distributions at acoustic frequencies between 0.5 and $3 \mathrm{MHz}$. The Journal of the Acoustical Society of America, 67(1): 135-146. https://doi.org/10.1121/1.384472

[13] Hwang, B.K., Furusawa, M., Ogata, M. (2007) Validation of multi-frequency inversion method by using dummy scatterers of zooplankton. Fisheries Science, 73(2): 250-262. https://doi.org/10.1111/j.14442906.2007.01331.x

[14] Blanc, S., Mosto, P., Benitez, C., Milou, M., Lascalea, G. (1998). Acoustical response of phytoplankton volume scatterers at ultrasonic frequencies as an indicator of pollution in sea waters. Transactions on Ecology and the Environment 18 : 231-240. https://doi.org/10.2495/CENV980221

[15] Bok, T.H., Na, J., Paeng, D.G. (2013). Diel variation in high-frequency acoustic backscatter from Cochlodinium polykrikoides. The Journal of the Acoustical Society of America, 134(2): $\quad$ EL140-EL146. https://doi.org/10.1121/1.4812437

[16] Kim, J., Choi, J.W., Kang, D. (2012). Laboratory experiment to measure $5-\mathrm{MHz}$ volume backscattering strengths from Red-tide causing microalgae Chattonella antiqua. Ocean Science Journal, 47(3): 173-179. https://doi.org/10.1007/s12601-012-0017-3

[17] Bok, T.H., Paeng, D.G., Kim, E., Na, J., Kang, D. (2010). Ultrasound backscattered power from Cochlodinium polykrikoides, the main red tide species in the Southern Sea of Korea. Journal of Plankton Research, 32(4): 503-
514. https://doi.org/10.1093/plankt/fbq001

[18] Tang, D., Di, B., Wei, G., Ni, I.H., Oh, I.S., Wang, S. (2006). Spatial, seasonal and species variations of harmful algal blooms in the South Yellow Sea and East China Sea. Hydrobiologia, 568(1): 245-253. https://doi.org/10.1007/s10750-006-0108-1

[19] Hay, A.E. (1983). On the remote acoustic detection of suspended sediment at long wavelengths. Journal of Geophysical Research, 88(C12): 7525. https://doi.org/10.1029/jc088ic12p07525

[20] Morse, P.M. (1948). Vibration and Sound. Second edition, 468 pages, illustrations, $15 \times 23 \mathrm{~cm}$. New York, McGraw-Hill Book Co., Inc. Journal of the Franklin Institute, 246(5): 434. https://doi.org/10.1016/00160032(48)90364-0

[21] Liu, R.Y. (2008). Checklist of marine biota of China seas. Science Press, Academia Sinica, Beijing. 175-186.

[22] Guillard, R.R.L., Ryther, J.H. (1962). Studies of marine planktonic diatoms: I. Cyclotella nana Hustedt and Detonula confervacea (CLEVE) Gran. Canadian Journal of $\quad$ Microbiology, $8(2)$ : $229-239$. https://doi.org/10.1139/m62-029

[23] Guo, H., Lin, F.A., Liu, Y.J., Xu, D.Y., Lu, X.W. (2014). High-incidence HABs causative species in China coastal waters and the forewarning method based on the HABs Risk Index. Marine Environmental Science, 1: 94-98.

[24] Jiang, T.J., Tong, M.M., Qi, Y.Z. (2006). Colors for early warning of harmful algal blooms and hazard classification and grading. Acta Ecologica Sinica, 26(6): 2035-2040. https://doi.org/10.3321/j.issn:10000933.2006.06.051

[25] Gluschke, M., Wellmitz, J., Lepom, P. (2004). A case study in the practical estimation of measurement uncertainty. Accreditation and Quality Assurance, 10(3): 107-111. https://doi.org/10.1007/s00769-004-0895-x 